\title{
Erratum to: Clinical trial and in-vitro study comparing the efficacy of treating bony lesions with allografts versus synthetic or highly-processed xenogeneic bone grafts
}

Eva Johanna Kubosch ${ }^{1}$, Anke Bernstein ${ }^{1}$, Laura Wolf ${ }^{1}$, Tobias Fretwurst ${ }^{2}$, Katja Nelson ${ }^{2}$ and Hagen Schmal ${ }^{1,3,4^{*}}$

\section{Erratum}

After publication of the original article [1], the authors noticed an error in the 'Methods' section. Within the 'Patients' sub-section, the first item in the list of synthetic or highly processed xenogeneic bone substitutes (SBS) should have been given as Orthoss $^{\circ}$, rather than BioOss ${ }^{\circ}$. The sentence should therefore have read as follows:

"The following SBS were used: Orthoss ${ }^{\circ}$ (Geistlich, Wolhusen, Switzerland) in $41.4 \%$, and at clearly lower percentages, respectively: Norian SRS ${ }^{\odot}$ (Synthes) (8.6\%), Chronos $^{\circ}$ (Synthes) (1.7\%), Atlantik ${ }^{\circ}$ (Argomedical) $(2.6 \%)$, Alaska $^{\circ}$ (Argomedical) (0.9\%), Endobone ${ }^{\circ}$ (Biomet) $(4.3 \%$ ), Pyrost $^{\circ}$ (Stryker) (0.9 \%), Nanostim ${ }^{\circ}$ (Medtronic) (37.1\%), Actifuse $^{\oplus}$ (Baxter) $(0.9 \%)$, Tutobone ${ }^{\circ}$ (Novomedics) (0.9\%) and PerOssal ${ }^{\bullet}$ (Botiss) (0.9\%)."

\section{Author details}

'Department of Orthopedics and Trauma Surgery, Albert-Ludwigs University Medical Center, Freiburg, Germany. ${ }^{2}$ Department of Craniomaxillofacial Surgery, Albert-Ludwigs University Medical Center, Freiburg, Germany.

${ }^{3}$ Department of Orthopaedics and Traumatology, Odense University Hospital, Odense, Denmark. ${ }^{4}$ Department of Clinical Research, University of Southern

Denmark, Odense, Denmark.

\section{Published online: 09 March 2016}

\section{Reference}

1. Kubosch EJ, Bernstein A, Wolf L, Fretwurst T, Nelson K, Schmal H. Clinical trial and in-vitro study comparing the efficacy of treating bony lesions with allografts versus synthetic or highlyprocessed xenogeneic bone grafts. BMC Musc Disord. 2016;17:77.

\footnotetext{
* Correspondence: hagen.schmal@freenet.de

'Department of Orthopedics and Trauma Surgery, Albert-Ludwigs University Medical Center, Freiburg, Germany

${ }^{3}$ Department of Orthopaedics and Traumatology, Odense University Hospital, Odense, Denmark
} 